\title{
Editorial:
}

\section{Undergraduate medical education: where are we?}

Medical care is essential for ensuring effective existence of the individual as well as the society. Irrespective of the nature of aims and priorities of the society, ensuring the health of the human resources is essentially an automatic priority of highest value. Medical and health care are highly personalized services and should cater to the individual needs which are unique and has a wide spectrum of activities. No country is in a position to provide the services in full measure. A hierarchy of facilities of graded complexity which are linked together functionally is the partial but practical answer to the problem.

The personnel rendering the services are, in turn, of varying background education. They have to be knit together as a team. Traditionally, medical doctor is designated as teamleader but doctors are not specifically trained in administrative and managerial aspects of health care. When, ultimately efforts are made to train them at a later date, after graduation, they do not evince sufficient interest to the extent that they become less amenable to be trained.

The stated aim of undergraduate medical education is to contribute toward the training of a non-specialized physician (general or basic) whose formation is in keeping with the health needs of the country, the evolution of medical science; and the requirements springing from the nature and responsibility of the physician's functions and his position in society.

Medical education by itself is a subculture. Like the culture of the humanity in general, it evolves. At macrolevel, the responses of the humanity to problems encountered are limited in nature but there is enormous scope for refining the response. So, the evolution occurs in a spiral fashion. But the question to be answered is whether this is an upward spiral or downward spiral or a horizontal spiral which, in effect, amounts to going in circles without a real change! One of the profound changes that the medical education has to contend with, at present, is rapid transition from labour intensive profession to capital and technology intensive industry. ${ }^{1,2}$

As for the curriculum, insistence on uniformity of syllabus and stereotyped framework for Institutions at varying degrees of development is a retrograde step and smacks of lack of imagination. This fosters mediocrity and kills the instinct to innovate. The education of the health care professions and the development of the health care services and scientific research are intertwined. Hence, while planning the curriculum for the medical education there is need to focus attention beyond the current level of development in the regional health care services and simultaneously bring changes in the structure and function of the health care institutions such that trained personnel can apply as much of their knowledge and skills as possible for the benefit of the community at large. ${ }^{3}$ This is imperative considering the fact that the government contributes greater part of the expenditure for the health of the community. However, the high cost of quality medical education makes it ineffective to plan for all centers to meet immediately the same standards as those required for the national institutes of excellence. As a part of the medical education policy, focus should be on practical and operational aspects of use of existing knowledge. This effectively serves to strengthen the linkage between teaching Institutions and other health care institutions in the health care delivery system. However, such coordination is hard to find anywhere.

Analogous to the principle of primary health care as the underlying principle for "health for all," we have to think of primary medical education that addresses the concerns of the Society in a spirit of self determination and self sufficiency. Too much stress on minutiae and details leads us to nowhere and the young students get disenchanted with the whole system of medical education. They are spending a major part of their 
formative period in one medical institution and their future is made or marred there itself. Medical teachers as part of the institution are solely responsible to shape their future.

The education of the future basic doctor has to be on a strong scientific and social foundation. ${ }^{3}$ However, the present system of medical education is totally divorced from these aims. The student selection is based on the power of cramming than anything else. By the time student joins the medical institution, he/she is already drained of energy and there is need to rejuvenate. The whole gamut of basic sciences has to be mastered in first year of their study in a new and strange milieu. Admissions drag on for a month and a preparation time of at least one month is given prior to university examinations. In addition, there is provision for vacation of one month. This leaves us with an effective training period of nine months. However, this gets truncated due to days spent on three formative assessment tests and public holidays. This leaves students with a period of about 8 months to attend lectures, practical sessions including record work and to study and assimilate the subject matter and then prepare for the examination. The practical and vivavoce which are introduced for the first time in their educational career are too stressful for them to cope up with. ${ }^{4}$ It is shocking to know that most students spend, on average, three months for anatomy, two months for physiology and one month for biochemistry. One can imagine what type of an edifice can be built on such a shaky foundation. It is not difficult to surmise what happens afterwards when they pursue their clinical studies from $3^{\text {rd }}$ semester to $9^{\text {th }}$ semester. The scheme for implementation of the clerkship for inculcating clinical skill and mentoring for fostering appropriate attitude and desirable behavioural traits for the doctor in making are far from satisfactory save for a few exceptions. The difficulties of the young student are so much compounded that only those with strong mental fibre are able to get along unscathed.

The other and more important aspect of medical education is the actual teaching. If the student has abiding faith in his teacher, he/she can learn steadily and at an accelerated pace. Analogous to the aphorism that "when everything fails, doctor is the medicine", it can rightly be stated that "when everything is amiss, a good medical teacher is the education", as the power of the teacher as a role model is enormous. While a few have innate talent in teaching and communicating, majority of medical teachers have to be 'trained teachers' or lay teachers. Traditionally, the medical teachers either assume the role of a teacher or it is ascribed to them without reference to the fact of their being specially trained for the purpose. The assumption is that a doctor with a postgraduate medical qualification automatically qualifies as a medical teacher. It is fallacious as there is no systematic training in pedagogy in most of the medical colleges. Only for the past few years systematic efforts have been in place for at least orienting the medical teachers in the nuances of medical education and content and methods of teaching. This aspect of medical education is much neglected as it is considered as domain of only Medical Education Technology Unit. The principles underlying the mechanics of adult teaching-learning process are utterly neglected both by the teacher and the taught. This deprives both the teacher and the student of the sense of mastery and self fulfillment. Medical Council of India (MCI) regulations specify that two thirds of the teaching hours should be spent for non-didactic teaching. ${ }^{5,6}$ This aspect varies from Institution to Institution and the experience needs to be shared and explored for better transaction of curriculum in this arena. Inspite of all the constraints, it has to be ensured that each student gains mastery over basic and essential aspects of practical medical and health care of the people. With large number of admissions, the identity of the students is getting dissolved and the stated goal is becoming more and more difficult to achieve and maintain.

A basic doctor is supposed to cater to the health needs of the population of the region. As majority of our people reside in rural areas, the student needs to understand the nuances to fulfilling their aspirations. 
Training of students with rural orientation has always been rightly stressed by politicians and policy makers. But the irony is that no worthwhile effort has been put to plan and effectively carry out training in rural milieu. Shortages of qualified personnel with appropriate motivation and issue of logistics have always remained as unsolved problems. Central and state government orders and circulars and budget allocations generally ignore the Rural Health Training Centres which are supposed to be the training ground for future Medical Officers of Primary Health Centres and Community Health Centres for which substantial budget allocations are made. If only the Reorientation of Medical Education (ROME) Programme launched in 1977 had properly been implemented and continued, all health care facilities of a region must have come under the direct influence and direction of Medical Colleges of the region by now. ${ }^{7}$ Unfortunately, it has always been the story of missed opportunity.

The formation of Universities of Health Sciences and proliferation of institutions of Health Profession has added another dimension to the constraints of medical education. In the name of safeguarding the standards of education, senior faculty members are often drafted for supervision, observership, administrative meetings and inspections. In addition, the services of Medical teachers are often requisitioned for training of other cadres of personnel whose educational standards are governed by university rules and rules of the respective Councils. The inevitable consequence is inability of the medical teachers to maintain undivided attention to their tasks of training medical students. While the importance of medical college, as a part of the general university, in training a humanistic doctor was recognized in the past by many, now the specialization and subspecialization in a profession oriented University are ruling the roost and general public is caught in the whirlpool of explosion of knowledge dissemination and specialization culture and lack of financial resources to meet the expenditure for extensive, exhaustive and specialized care. In the summative assessment, adoption of newer methods like Objective Structured Clinical Examination (OSCE) and bedside rounds in some Institutions appear to be so defective that the remedy appears to be worse than the malady.

Other equally important aspects of education in general are maintenance of complete health and shaping of the personality of the individual student by participation in extracurricular activities and inculcating the habit of conducting oneself ethically. For this, full fledged facilities need to be created and tokenism will not help in any manner. This and participation in other group activities fosters buddy feeling. Formal shaping of buddy system as a source of strength needs to be fully explored. Just as treating doctor is the advocate for the patient, the Medical teacher should become an effective advocate for the student. The MCI and governments also stress on this aspect but implementation is far from satisfactory.

While solution to all the questions cannot be found instantaneously, it is important to recognize whether problems exist, whether they are real or imaginary and intellectualized, whether they are personal or Institutional and who are all the people responsible to solve them and how they can be solved effectively. In this quest let it be recognized that "the old order changeth yielding place to new, lest one good custom should corrupt the world" but let it also be ensured that "the clear stream of reason does not lose its way into the dreary desert sand of dead habit".

Organization of vibrant and effectively functioning Medical Education Technology Unit, thus, becomes a very important component of medical education system. ${ }^{8}$ This unit should shoulder the onerous responsibility of inculcating desirable attributes and characteristics of 'social physician ' so that the pristine position of the doctor is preserved in the society.

We have to rely on our ancient wisdom of 'parasparam bhavayantah' - let us help one another and also ensure that 'Sahanavavatu, sahanoubhunaktu, sahaveeryamkaravavahai, Tejaswinavadhitamastu, 
ma vidvishavahai'- May we (the teacher and the taught) together acquire the capacity for knowledge. These are the first steps to ensure Health for All in the spirit of

Sarve santu sukhinah,

Sarve santu niramayaha,

Sarve bhadrani pasyantu,

Ma kaschid dukha-bhag bhavet.

Can we hope that the present generation of teachers will live up to the task? Hopefully, yes!

M.S.Sridhar

Principal,

Sri Venkateswara Medical College,

Tirupati

e-mail: sridhar.ms68@gmail.com

Received: 23 July, 2013.

Sridhar MS. Undergraduate medical education: where are we? J Clin Sci Res 2013;2:193-6.

\section{REFERENCES}

1. Supe A, Burdick WP. Challenges and issues in medical education in India. Acad Med 2006;81:1076-80.

2. Ghosh K, Ross C. The teacher and the taught: medical education in India at the crossroads. Natl Med J India 2009;22:147-9.

3. Jayakrishnan T, Honhar M, Jolly GP, Abraham J, T J. Medical education in India: time to make some changes. Natl Med J India 2012;25:164-7.

4. Holla SJ, Ramachandran K, Isaac B, Koshy S. Anatomy education in a changing medical curriculum in India: medical student feedback on duration and emphasis of gross anatomy teaching. Anat Sci Educ 2009;2:179-83.

5. Medical Council of India. Regulation of Graduate Medical Education. 1997. Notification. Gazette of India, part III, sect. 4, May 17, 1997.

6. Vision 2015. Medical Council of India. Available at URL: http://www.mciindia.org/tools/announcement/ MCI_booklet.pdf. Accessed on July 24, 2013.

7. Poulose KP, Natarajan PK. Re-orientation of medical education in India past, present and future. Indian J Public Health 1989;33:55-8.

8. Adkoli BV, Sood R. Faculty development and medical education units in India: a survey. Natl Med J India 2009;22:2832. 